\title{
Irregular Polar Coding for Multi-Level Modulation in Complexity-Constrained Lightwave Systems
}

\author{
Toshiaki Koike-Akino ${ }^{1}$, Congzhe Cao ${ }^{1,2}$, Ye Wang ${ }^{1}$, Stark C. Draper ${ }^{3}$, David S. Millar ${ }^{1}$, Kieran Parsons ${ }^{1}$, \\ Keisuke Kojima ${ }^{1}$, Milutin Pajovic ${ }^{1}$, Lidia Galdino ${ }^{4}$, Daniel J. Elson ${ }^{4}$, Domaniç Lavery ${ }^{4}$, Polina Bayvel ${ }^{4}$ \\ ${ }^{1}$ Mitsubishi Electric Research Laboratories (MERL), Cambridge, MA 02139, USA. koike@merl.com \\ 2 Dept. of Electrical \& Computer Engineering, University of Alberta, Edmonton, AB T6G 2V4, Canada. \\ ${ }^{3}$ Electrical \& Computer Engineering, University of Toronto, Toronto, ON M5S 3G4, Canada. \\ ${ }^{4}$ Optical Networks Group, University College London (UCL), Torrington Place, London, WC1E 7JE, UK.
}

Abstract We introduce novel irregular polar codes, which extend the regular polar coding by flexible polarization pruning. We experimentally demonstrate that the proposed polar codes outperform state-ofthe-art LDPC codes, while the computational complexity in encoding and decoding can be significantly reduced by at least $30 \%$ over the regular polar codes with a marginal performance improvement.

\section{Introduction}

Multi-level modulation schemes with capacityapproaching forward error correction (FEC) based on low-density parity-check (LDPC) codes $^{1-4}$ have made a great contribution to increase data rates of coherent optical transceivers achieving beyond $\mathrm{Tb} / \mathrm{s}^{5-7}$. However, pursuit of high FEC performance has led to a significant increase in power consumption and circuit size. Hence, a good trade-off between performance and computational complexity is of importance. This paper introduces reduced-complexity polar codes as potential alternatives to LDPC codes.

Polar codes ${ }^{8-11}$ have drawn much attention in the coding theory community since their ability to achieve capacity over arbitrary discreteinput memoryless channel was proven in 2009. The recent breakthrough in algorithm development of successive cancellation (SC) list decoding ${ }^{11}$ has shown that polar codes can outperform state-of-the-art LDPC codes, in particular for low-complexity and latency-constrained systems ${ }^{10}$. Thus, polar codes are now considered as strong alternative candidates to LDPC codes in fifth-generation wireless standards.

In this paper, we propose a new family of polar codes, whose polarization units are irregularly pruned to drastically reduce the computational complexity. For LDPC codes, it is wellknown that irregular codes with specific degree distributions outperform regular ones. We show that the irregular polar codes can also improve the performance, yet realize complexity reduction by at least $30 \%$ in encoding and decoding. Experimental validation of the proposed irregular polar codes is also demonstrated over $400 \mathrm{~km}$

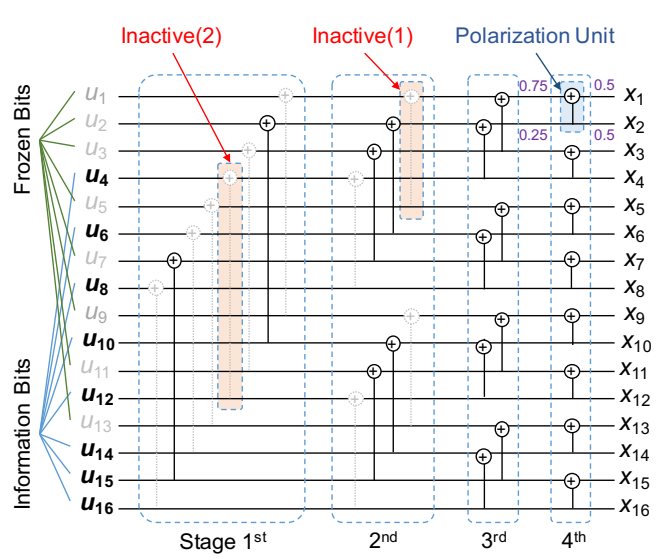

Fig. 1: Example of $(16,8)$ irregular polar codes having 10 inactivations over 32 polarization units.

transmission of Corning ${ }^{\circledR}$ Vascade ${ }^{\circledR}$ EX2000 fiber, for two $60 \mathrm{GBd}$ subchannels of dualpolarization 64-ary quadrature-amplitude modulation (DP-64QAM) with full C-band loading ${ }^{5}$.

\section{Irregular Polar Coding}

For an $(N, k)$ polar coding, there are $\log _{2}(N)$ polarization stages, each of which contains $N / 2$ polarization units, as depicted in Fig. 1 for $N=16$. Polar codes exploit so-called polarization phenomenon, where each polarization unit provides degraded and improved reliability. For example, when the code bits $x_{1}$ and $x_{2}$ have uniform reliability having erasure rate (Bhattacharyya parameter) of 0.5 , the upper branch of the polarization unit becomes unreliable with erasure rate of 0.75 , whereas the lower branch improves the reliability to 0.25 . The conventional polar codes have no flexibility in coding architecture except in the location of frozen bits. To increase the degree of freedom, we propose a new family called irregular polar coding where some of the polarization units are inactivated. Fig. 1 is an example having 


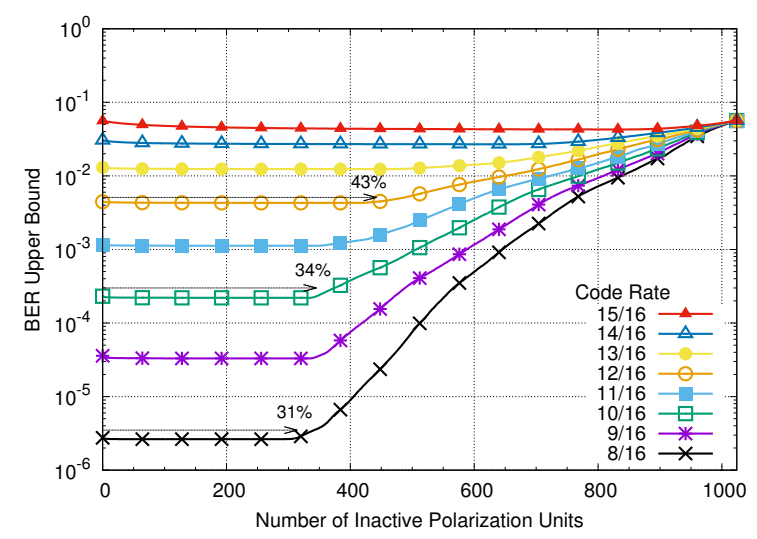

(a) High rates $R \geq 0.5$ at $4 \mathrm{~dB}$ SNR

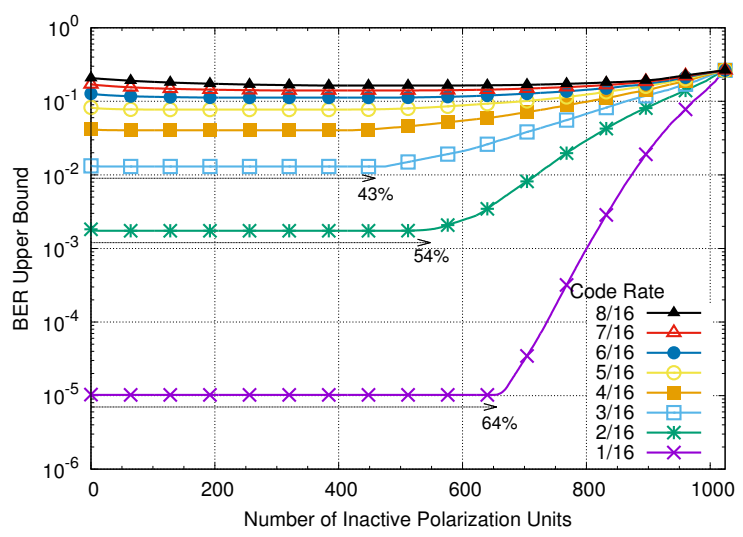

(b) Low rates $R \leq 0.5$ at $-4 \mathrm{~dB}$ SNR

Fig. 2: BER upper bound of irregular polar codes with $N=256$ for different code rates $R \in\{1 / 16,2 / 16, \ldots, 15 / 16\}$.

10 inactivations among 32 polarization units.

The key benefit of such inactivations is twofold: i) complexity reduction in encoding/decoding computations, and ii) potential performance improvement by adjusting weight distribution. For the code in Fig. 1, we can achieve 10/32 $31 \%$ complexity reduction since no computation is required at all for inactivated units in both encoding and decoding. Note that careful choice of polarization units to be inactivated cause no performance penalty. For example, the bit-error rate (BER) does not depend on the inactive unit (1) in this figure because no information bits only frozen bits are involved in the polarization. More importantly, the BER of this irregular code is slightly better than the regular one. This is because the inactive unit (2) does not involve frozen bits and the least-reliable information bit $u_{4}$ can be improved by pruning polarization to avoid degrading it.

We use a greedy design method for joint optimization of frozen bit and inactivated polarization locations, so that BER upper bound is minimized. Letting $Z_{i}$ be the Bhattacharrya parameter of the $i$ th information bit, the upper bound ${ }^{8}$ is expressed as $\frac{1}{k}\left(1-\prod_{i=1}^{k}\left(1-Z_{i}\right)\right)$. Figs. 2(a) and (b) show BER upper bound versus the number of inactivated polarization units for various code rates of $N=256$. Note that the number of inactive units $N_{\text {inact }}=0$ corresponds to the conventional regular polar codes, whereas $N_{\text {inact }}=1024$ corresponds to uncoded case. It is confirmed that our proposed irregular polar codes can reduce computational complexity by at least $31 \%$ with a little improvement in the upper bound. The irregular polar codes are more advantageous for code rates both higher and lower than 0.5 . It is because rate- $1 / 2$ codes have the most complicated interactions between frozen bits and information bits.

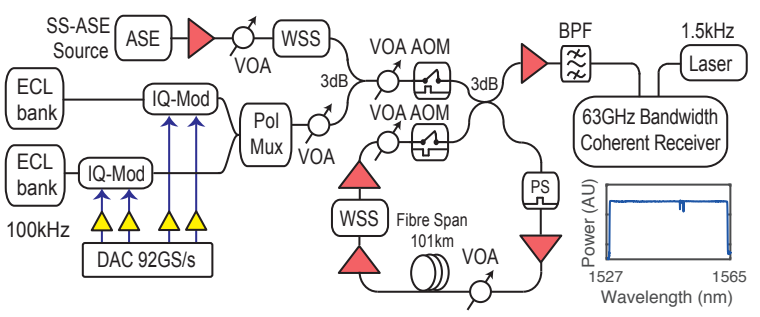

Fig. 3: Experimental setup ${ }^{5}$ for dual-carrier $60 \mathrm{GBd}$ DP-64QAM. Full C-band loading is emulated by ASE $^{12}$.

\section{Experimental Validation}

For performance validation of our irregular polar codes, we use the experimental setup of the dual-carrier 60 GBd DP-64QAM transmission ${ }^{5}$, whose schematic is shown in Fig. 3. The experimental setup and receiver digital signal processing (DSP) were of the same structure to that described in our previous work ${ }^{5}$. Two external cavity lasers (ECLs) with $100 \mathrm{kHz}$ nominal linewidth and $62 \mathrm{GHz}$ spacing were used as carriers for two independent I/Q modulators. Full C-band loading was emulated by spectrally shaped amplified spontaneous emission (ASE) noise ${ }^{12}$. We used a training sequence followed by pilot-aided equalization with a pilot overhead of $1.43 \%$.

In our previous experiments ${ }^{5}$, a $1 \mathrm{~Tb} / \mathrm{s}$ transmission was demonstrated using a high-power LDPC code with very long codeword of 52,800 bits for a code rate of 0.71 . Because our scope of this paper is to consider low-power and lowlatency systems, we focus on relatively short FEC codes with $N \in\{256,1024,4096\}$. We compare irregular polar codes (having $33 \%, 37 \%$, and $31 \%$ inactivations of polarization units, respectively, for $N=256,1024$, and 4096) with state-of-the-art Pareto-optimal LDPC codes $^{4}$ (whose analytical threshold in infinite lengths is within $0.5 \mathrm{~dB}$ from Shannon limit) for a code rate of 0.625. Random interleaving was carried out across all bit 


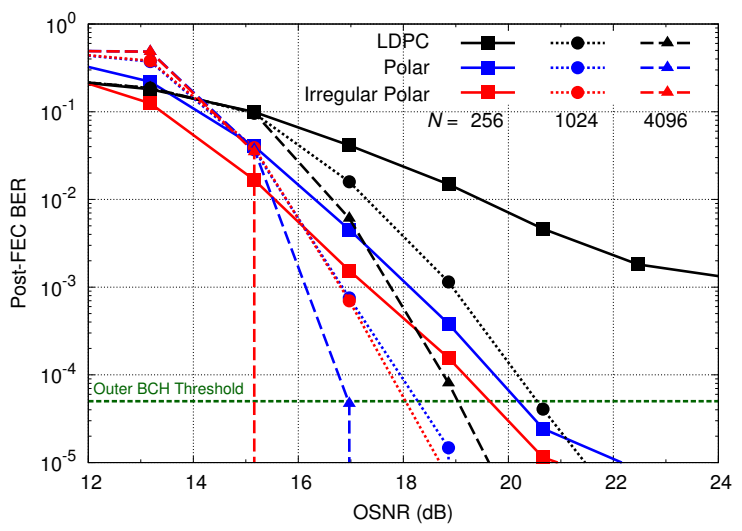

Fig. 4: Experimental BER in back-to-back configuration.

positions, polarizations and wavelength subchannels although more practical interleavers suited for polar codes were investigated ${ }^{9,10}$. The LDPC decoder used the layered sum-product algorithm with 8 iterations, whereas the polar decoder used SC list decoding with 8-bit cyclic-redundancy check (CRC) and 32 lists. An outer hard-decision high-rate $\mathrm{BCH}$ code $^{5}$ (threshold is $5 \times 10^{-5}$ ) is assumed to achieve a BER below $10^{-15}$.

The back-to-back results are shown in Fig. 4, which verified that the polar codes outperform LDPC codes. This is because LDPC codes do not perform well for short word lengths and limited number of iterations ${ }^{10}$. Our irregular polar codes can also offer improved BER against regular polar codes. Specifically, the measurement at an optical signal-to-noise ratio (OSNR) above $17 \mathrm{~dB}$ had no errors with irregular polar coding of $N=4096$ over the approximately $3 \times 10^{6}$ bits in a single capture, whereas errors were observed for regular polar codes. The results after transmission over $300 \mathrm{~km}$ and $400 \mathrm{~km}$ are shown in Figs. 5 and 6 , which demonstrated that the system margin can be significantly improved by the polar coding compared to LDPC codes.

\section{Conclusions}

We proposed novel irregular polar codes, which achieve both performance improvement and complexity reduction by at least $30 \%$. We experimentally demonstrated that the proposed polar codes can outperform state-of-the-art LDPC codes.

\section{Acknowledgements}

This work was in part funded by the UK EPSRC Programme Grant UNLOC EP/J017582/1. We would like to thank all researchers ${ }^{5}$ involved in experiments.

\section{References}

[1] I. B. Djordjevic, "Advanced coded-modulation for ultrahigh-speed optical transmission," OFC (2014): W3J-4.

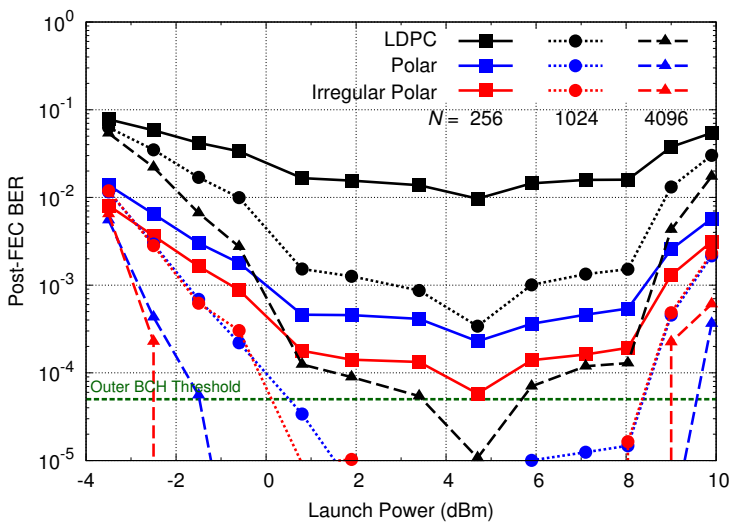

Fig. 5: Experimental BER in $300 \mathrm{~km}$ transmission.

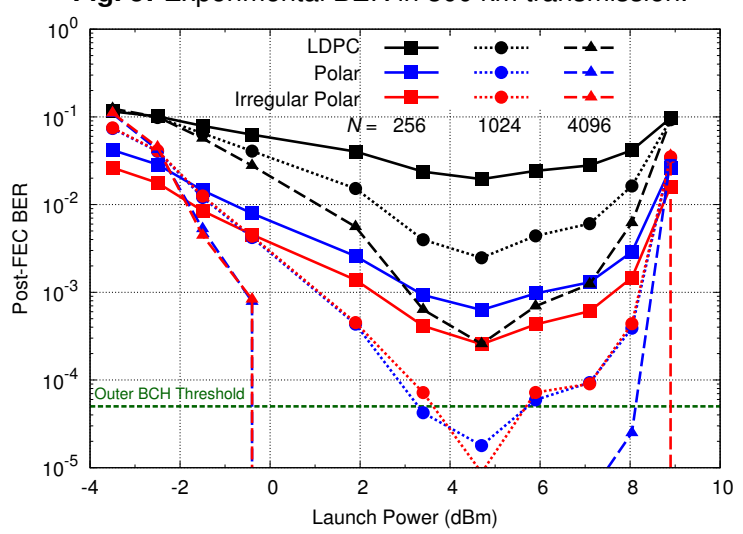

Fig. 6: Experimental BER in $400 \mathrm{~km}$ transmission.

[2] L. Schmalen et al., "Spatially coupled soft-decision error correction for future lightwave systems," JLT 335 (2015): 1109-1116.

[3] A. Leven, L. Schmalen, "Status and recent advances on forward error correction technologies for lightwave systems," JLT 3216 (2014): 2735-2750.

[4] T. Koike-Akino et al., "Iteration-aware LDPC code design for low-power optical communications," JLT 342 (2016).

[5] D. S. Millar et al., "A simplified dual-carrier DP-64QAM 1 Tb/s transceiver," OFC (2017): M3D-2.

[6] R. Maher et al., "Increasing the information rates of optical communications via coded modulation: a study of transceiver performance," Nat. Sci. Rep. 621278 (2016).

[7] G. Raybon et al., "Single carrier high symbol rate transmitter for data rates up to $1.0 \mathrm{~Tb} / \mathrm{s}$," OFC (2016): Th3A2.

[8] E. Arıkan, "Channel polarization: A method for constructing capacity-achieving codes for symmetric binary-input memoryless channels," IEEE TIT 557 (2009).

[9] D. M. Shin, S. C. Lim, K. Yang, "Mapping selection and code construction for $2^{m}$-ary polar-coded modulation," IEEE COMML 166 (2012): 905-908.

[10] T. Koike-Akino et al., "Bit-interleaved polar-coded modulation for short-block optical transmission," OFC (2017): W1J6.

[11] I. Tal, A. Vardy, "List decoding of polar codes," IEEE TIT 615 (2015): 2213-2226.

[12] D. J. Elson et al., "High spectral density transmission emulation using amplified spontaneous emission noise," Opt. Lett. 41 (2016): 68-71. 